\title{
A network meta-analysis: the overall and progression-free survival of glioma patients treated by different chemotherapeutic interventions combined with radiation therapy (RT)
}

\author{
Ling $\mathbf{Q i}^{1, *}$, Lijuan Ding ${ }^{2, *}$, Shuran Wang ${ }^{3}$, Yue Zhong ${ }^{3}$, Donghai Zhao ${ }^{3}$, Ling Gao ${ }^{2}$, \\ Weiyao Wang ${ }^{1}$, Peng Lv ${ }^{1}$, Ye $X u^{1}$ and Shudong Wang ${ }^{4}$ \\ ${ }^{1}$ Basic Medical College, Jilin Medical University, Jilin, China \\ 2 Department of Radiation Oncology, First Hospital of Jilin University, Changchun, China \\ ${ }^{3}$ Department of Science and Technology, Jilin Medical University, Jilin, China \\ ${ }^{4}$ Center of Cardiovascular Diseases, First Hospital of Jilin University, Changchun, China \\ * These authors have contributed equally to this work \\ Correspondence to: Ye Xu, email: drzhhaiquan@yeah.net
}

Shudong Wang, email: liqhli@126.com

Keywords: chemotherapy, radiotherapy, glioma, network meta-analysis, temozolomide

Received: April 05, $2016 \quad$ Accepted: July 10, $2016 \quad$ Published: July 21, 2016

\section{ABSTRACT}

Different chemotherapy drugs are generally introduced in clinical practices combining with therapy for glioma treatment. However, these chemotherapy drugs have rarely been compared with each other and the optimum drug still remains to be proved. In this research, medical databases were consulted, PubMed, Embase and Cochrane Library included. As primary outcomes, hazard ratio (HR) of overall survival (OS) and progression-free survival (PFS) with their corresponding $95 \%$ credential intervals ( $\mathrm{CrI}$ ) were reported. A network meta-analysis was conducted; the surface under the cumulative ranking curve (SUCRA) was utilized for treatment rank and a cluster analysis based on SUCRA values was performed. This research includes 14 trials with 3,681 subjects and eight interventions. In terms of network metaanalysis, placebo was proved to be inferior to the combination of temozolomide (TMZ), nimustine (ACNU) and cisplatin (CDDP). Also, bevacizumab (BEV) in conjunction with TMZ were significantly more effective than placebo with an HR of 0.40 . The estimated probabilities from SUCRA verified the above outcomes, confirming that the combination of TMZ, ACNU and CDDP exhibited the highest ranking probability of 0.889 with respect to OS, while BEV in combination with TMZ - with a probability of 0.772 - ranked the first place with respect to PFS. According to the results of this network meta-analysis, the combination of (1) TMZ, ACNU and CDDP; (2) BEV in combination with $T M Z$ and (3) cilengitide in combination with $T M Z$, are considered as the preferable choices of chemotherapy drugs for glioma treatment.

\section{INTRODUCTION}

Glioma tumours developed from neoplastic glial cells - which provide support and protection for the peripheral and central nerve system [1] are a common type of primary brain tumours. This specific type of tumour accounts for approximately $30 \%$ of all brain and spine tumours and $80 \%$ of all malignant brain tumours [2]. As suggested by World Health Organization (WHO), gliomas can be further classified into four stages on the basis of their histopathological patterns, the presence of nuclear pleomorphism, the degree of increased mitotic activity and cellularity, endothelial cell proliferation and the degree of necrosis [4]. In stage I and II, gliomas are considered as low-risk cases in which more optimistic prognosis are usually observed whereas gliomas in stage III and IV are classified as high-risk cases and referred to as malignant tumours. For instance, anaplastic astrocytomas (AA) and 
glioblastomas (GB) are common types of stage III and IV glioma cases, and the five-year survival rate of GB patients is less than 3\% [5]. Another key factor for the prognosis of glioma patients is the onset age and a large number of studies have concluded that the average survival time of GB patients was negatively correlated to their ages at which GB was diagnosed [6].

Surgery, radiation therapy (RT) or chemotherapy is introduced when oncology is certified. Surgical approaches usually involve biopsy and resection for which the extent and timing are essential factors for prognosis. Numerous studies have indicated that the extensive or gross total surgery is correlated with a less recurrent incidence and longer survival time compared with the approach of limited surgery [7-9].. RT is usually conducted for HGG patients once surgeries had been introduced to these patients and it is also appropriate for some patients who are not eligible for surgeries. Local RT, three-dimensional conformal RT and stereotactic radiosurgery are three popular RT techniques introduced in clinical practices. Apart from that, brachytherapy, radiosurgery and hyper fractionation have been introduced in order to improve remedial outcomes [11]. For instance, temozolomide (TMZ), a DNA alkylating agent, has been introduced into RT and this approach doubled the two-year survival rate to approximately $27 \%$ compared to single RT [12].

Furthermore, anti-tumour alkylating agents including carmustine (BCNU), nimustine (ACNU), lomustine $(\mathrm{CCNU})$ and other nitrosourea agents also play important roles in glioma treatment. For recurrent GB, biological agents and monoclonal antibodies such as bevacizumab (BEV) are highly recommended in clinical practices [12]. Since single chemotherapy agent was limited in its effectiveness for suppressing tumour cells, two or more chemotherapy agents with synergistic effects have been introduced. Procarbazine, lomustine as well as vincristine (PCV) can be considered as commom choices. Although RT in conjunction with chemotherapy has enhanced the effectiveness of treatment, it is still challenging to determine the optimal combination of treatments due to the wide range of available interventions.

There are a large amount of studies comparing the prognosis of patients who had been treated with mono or combined anti-glioma drugs. However, the majority of evidence was generated by pair-wise meta-analyses in which only head-to-head trails were compared. Some results appeared to be biased due to the lack of study subjects. Others appeared to have contradictory results which were misleading in the current literature. This search enabled us to compared the overall survival (OS) and progression-freesurvival (PFS) status of glioma patients who had been treated with: (1) TMZ, (2) PCV, (3) BEV combined with TMZ, (4) Nimotuzumab, (5) Cilengitide combined with TMZ, (6) TMZ combined with ACNU and cisplatin (CDDP), (7) dibromodulcitol (DBD) combined with BCNU and (8) alpha-difluoromethylornithine (DMFO) combined with PCV.

\section{RESULTS}

\section{Included studies}

Fourteen eligible trials including 3,681 subjects were selected from 51 studies assessed in this meta-analysis [19-32]. Initially, 1,647 publications were identified by keywords searching in three mentioned medical databases and 41 of them are meta-analysis. Another 69 metaanalyses were found and included through reviewing

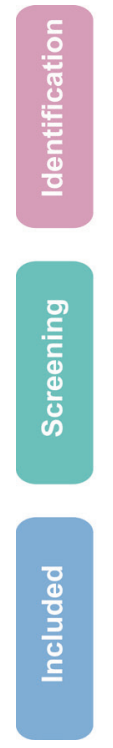

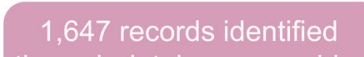

through database searching

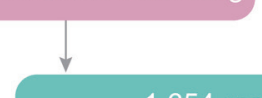

1,654 records identified

and 763 duplicates removed

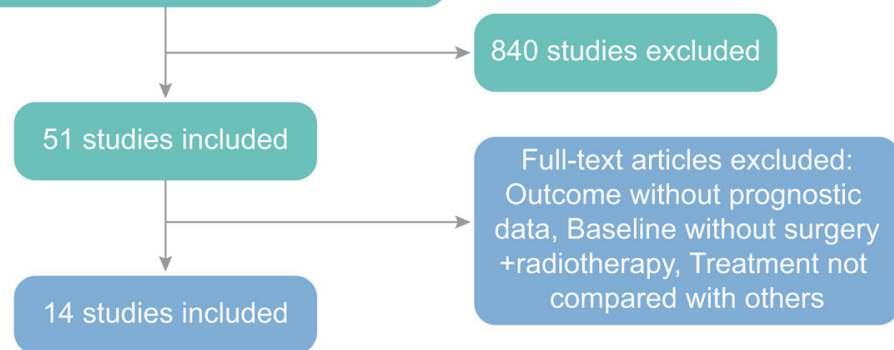

Figure 1: Giloma flow diagram on sampling of meta-analysis. 
Table 1: Main characteristics of included studies.

\begin{tabular}{|c|c|c|c|c|c|c|c|c|c|c|c|c|}
\hline \multirow{2}{*}{ Author } & \multirow{2}{*}{ Year } & \multirow{2}{*}{ Country } & \multirow{2}{*}{ Disease } & \multirow{2}{*}{ Situation } & \multirow{2}{*}{ Surgery } & \multirow{2}{*}{ Radiotherapy } & \multicolumn{2}{|c|}{\begin{tabular}{|l|}
$\begin{array}{l}\text { Experimental } \\
\text { Group }\end{array}$ \\
\end{tabular}} & \multicolumn{2}{|c|}{\begin{tabular}{|l|} 
Control \\
Group
\end{tabular}} & \multirow{2}{*}{$\begin{array}{l}\text { OS } \\
\text { (HR and } 95 \% \mathrm{CI})\end{array}$} & \multirow{2}{*}{$\begin{array}{l}\text { PFS } \\
\text { (HR and 95\%CI) }\end{array}$} \\
\hline & & & & & & & Size & Drugs & Size & Drugs* & & \\
\hline Solomon & 2013 & Cuba & HGG & $-\#$ & Biopsy/Resection & $\sqrt{ }$ & 38 & E & 32 & A & $0.68(0.42,1.11)$ & $0.75(0.49,1.16)$ \\
\hline Stupp & 2005 & Switzerland & GB & Newly & Biopsy/Resection & $\sqrt{ }$ & 287 & B & 286 & A & $0.63(0.52,0.75)$ & $0.54(0.45,0.64)$ \\
\hline van den Bent & 2006 & Netherland & $\mathrm{AO} / \mathrm{AOA}$ & - & Biopsy/Resection & $\sqrt{ }$ & 185 & C & 183 & A & $0.85(0.65,1.11)$ & $0.68(0.53,0.87)$ \\
\hline Shaw & 2012 & USA & LGG & - & Biopsy/Resection & $\sqrt{ }$ & 125 & C & 126 & A & $0.72(0.47,1.10)$ & $0.60(0.41,0.86)$ \\
\hline Chinot & 2014 & France & GB & Newly & Biopsy/Resection & $\sqrt{ }$ & 458 & $D$ & 463 & B & $0.88(0.76,1.02)$ & $0.64(0.55,0.74)$ \\
\hline Tham & 2013 & Australia & $\mathrm{AO} / \mathrm{AOA}$ & - & Biopsy/Resection & $\sqrt{ }$ & 36 & B & 26 & A & $1.03(0.50,2.11)$ & $1.29(0.71,2.33)$ \\
\hline Stupp & 2014 & Switzerland & GB & Newly & Biopsy/Resection & $\sqrt{ }$ & 272 & $\mathrm{~F}$ & 273 & B & $1.02(0.81,1.29)$ & $0.93(0.76,1.13)$ \\
\hline Kim & 2011 & Korea & GB & Newly & Biopsy/Resection & $\sqrt{ }$ & 40 & G & 42 & B & $0.52(0.24,1.12)$ & $0.89(0.49,1.62)$ \\
\hline Hildebrand & 2008 & Belgium & $\mathrm{AO} / \mathrm{AOA}$ & Newly & Biopsy/Resection & $\sqrt{ }$ & 94 & $\mathrm{H}$ & 99 & A & $0.77(0.56,1.06)$ & $0.75(0.57,0.99)$ \\
\hline Levin & 2000 & USA & GBM & - & Biopsy/Resection & $\sqrt{ }$ & 134 & I & 138 & C & $1.00(0.90,1.10)$ & $1.00(0.90,1.10)$ \\
\hline Muni & 2010 & Italy & GB & Newly & Biopsy/Resection & $\sqrt{ }$ & 22 & B & 23 & A & $0.50(0.26,0.99)$ & $0.52(0.29,0.93)$ \\
\hline Kocher & 2008 & Germany & GB & - & Resection & $\sqrt{ }$ & 29 & B & 33 & A & $0.84(0.44,1.62)$ & $0.91(0.49,1.67)$ \\
\hline Nabors & 2015 & USA & GB & Newly & Biopsy/Resection & $\sqrt{ }$ & 88 & $\mathrm{~F}$ & 89 & B & $0.69(0.48,0.97)$ & $0.82(0.60,1.13)$ \\
\hline Combs & 2008 & Germany & $\mathrm{AO} / \mathrm{AOA}$ & - & Biopsy/Resection & $\sqrt{ }$ & 20 & B & 40 & A & $1.51(0.76,3.02)$ & $1.86(0.97,3.57)$ \\
\hline
\end{tabular}

HGG: High Grade Glioma (include GBM+AO/AOA); GB: Glioblastoma; AO/AOA: Anaplastic Oligodendroglioma/ Oligoastrocytomas; LGG: Low Grade Glioma; GBM: Glioblastoma Multiforme; HR: Hazard Ratio; OS, Overall Survival; PFS, Progression-free Survival; -, none reported.

* A: Placebo; B: TMZ; C: PCV; D: BEV+TMZ; E: Nimotuzumab; F: Cilengitide+TMZ; G: TMZ+ACNU+CDDP; H: DBD+BCNU; I: DMFO+PCV; TMZ: Temozolomide; PCV: Procarbazine, Lomustine and Vincristine; BEV: Bevacizumab; ACNU: Nimustine; CDDP: Cisplatin; DBD: Dibromodulcitol; BCNU: Carmustine; DMFO: Eflornithine;

references of relevant literature manually. Based on the content of abstracts and the full text of scientific papers, 51 studies were selected with 37 of the 51 studies excluded since interventions in these studies cannot form a closed loop. This resulted in the inclusion of 14 scientific papers that were published between 2000 and 2015. The entire process of literature search is illustrated in Figure 1. These 14 papers mentioned eight distinctive chemotherapy drugs that can be used for glioma: (1) TMZ, (2) PCV, (3) BEV in combination with TMZ, (4) Nimotuzumab, (5) Cilengitide in combination with TMZ, (6) TMZ combined with ACNU and CDDP, (7) DBD in combination with BCNU, and (8) DMFO in combination with PCV. Jadad scale of 14 included studies is summarized in Table S1.

\section{Characteristics of included trials}

The main characteristics and primary outcomes of the included studies and subjects are summarized in Table 1. The majority of selected studies focused on HGG patients who were either classified as GB, GBM or anaplastic oligodendroglioma/oligoastrocytomas (AA/ AOA). There were seven studies which included a total of 2,405 patients with $\mathrm{GB}$, while only one study investigated GBM with a total of 272 subjects. On the other hand, a randomized clinical trial with a sample of 251 LGG patients was included in our research. Besides that, only half of the 14 studies disclosed that whether glioma were newly formed cases whereas the other half of the studies failed to report this issue. All included patients have undergone radio therapeutic treatment and surgery. The entire network of comparisons among all eligible studies is demonstrated in Figure 2.

\section{Direct comparison}

The results of pair-wise comparisons were illustrated in Table 2. There appeared to be no significant difference in OS status between patients treated with placebo and those treated by TMZ, PCV, Nimotuzumab or DBD in conjunction with $\mathrm{BCNU}(\mathrm{HR}=0.78,95 \% \mathrm{CI}=0.56-1.11$; $\mathrm{HR}=0.81,95 \% \mathrm{CI}=0.65-1.02 ; \mathrm{HR}=0.68,95 \% \mathrm{CI}=$ $0.42-1.11 ; \mathrm{HR}=0.77,95 \% \mathrm{CI}=0.56-1.06)$. Also, there was no significant difference in OS status between patients treated with $\mathrm{TMZ}$ and those treated with $\mathrm{BEV}+\mathrm{TMZ}$, Cilengitide + TMZ or ACNU + CDDP + TMZ $(\mathrm{HR}=0.88$, $95 \% \mathrm{CI}=0.59-1.26 ; \mathrm{HR}=0.86,95 \% \mathrm{CI}=0.58-1.26 ; \mathrm{HR}=$ $0.52,95 \% \mathrm{CI}=0.24-1.12)$. Similarly, introducing DMFO into PCV did not improve the effectiveness of PCV significantly $(\mathrm{HR}=1.00,95 \% \mathrm{CI}=0.91-1.11)$.

Comparisons of PFS among different interventions were also listed in Table 2. PCV seemed to be the only 
Table 2: Pair-wise meta-analyses of direct comparisons between the eight drugs.

\begin{tabular}{|l|l|l|l|l|l|l|}
\hline Endpoints & Direct comparisons & $\boldsymbol{I}^{2}$ & Tau $^{2}$ & $\boldsymbol{P}_{\text {H }}$ values & HR $(\mathbf{9 5} \% \mathbf{C I})$ & $\boldsymbol{P}_{\text {HR }}$ values \\
\hline OS & TMZ vs. Placebo & $51.8 \%$ & 0.076 & 0.081 & $0.78(0.56,1.11)$ & 0.168 \\
\hline & PCV vs. Placebo & $0.00 \%$ & 0.000 & 0.517 & $0.81(0.65,1.02)$ & 0.070 \\
\hline & Nimotuzumab vs. Placebo & - & - & - & $0.68(0.42,1.11)$ & 0.120 \\
\hline & DBD+BCNU vs. Placebo & - & - & - & $0.77(0.56,1.06)$ & 0.108 \\
\hline & BEV+TMZ vs. TMZ & - & - & - & $0.88(0.76,1.02)$ & 0.089 \\
\hline & Cilengitide+TMZ vs. TMZ & $70.90 \%$ & 0.056 & 0.064 & $0.86(0.58,1.26)$ & 0.427 \\
\hline & TMZ+ACNU+CDDP vs. TMZ & - & - & - & $0.52(0.24,1.12)$ & 0.096 \\
\hline & DMFO+PCV vs. PCV & - & - & - & $1.00(0.91,1.11)$ & 1.000 \\
\hline & TMZ vs. Placebo & $80.90 \%$ & 0.242 & 0.000 & $0.87(0.53,1.43)$ & 0.581 \\
\hline & PCV vs. Placebo & $0.00 \%$ & 0.000 & 0.582 & $0.65(0.53,0.80)$ & 0.000 \\
\hline & Nimotuzumab vs. Placebo & - & - & - & $0.75(0.49,1.15)$ & 0.191 \\
\hline & DBD+BCNU vs. Placebo & - & - & - & $0.75(0.57,0.99)$ & 0.041 \\
\hline & BEV+TMZ vs. TMZ & - & - & - & $0.64(0.55,0.74)$ & 0.000 \\
\hline & Cilengitide+TMZ vs. TMZ & $0.00 \%$ & 0.000 & 0.523 & $0.90(0.76,1.06)$ & 0.217 \\
\hline & TMZ+ACNU+CDDP $v s$. TMZ & - & - & - & $0.89(0.49,1.62)$ & 0.702 \\
\hline & DMFO+PCV vs. PCV & - & - & - & $1.00(0.91,1.11)$ & 1.000 \\
\hline
\end{tabular}

*H: heterogeneity; HR: hazard ratio; CI: confidence interval; OS: overall survive; PFS: progression-free survival; TMZ: Temozolomide; PCV: Procarbazine, Lomustine, and Vincristine; DBD: Dibromodulcitol; BCNU: Carmustine; BEV: Bevacizumab; ACNU: Nimustine; CDDP: Cisplatin; DMFO: Eflornithine;

Table 3: The efficacy (overall survival and progression-free survival) of eight drugs in chemotherapy of glioblastoma followed by surgery and radiotherapy according to the network meta-analysis using hazard ratio (HR) and corresponding $95 \%$ credible intervals (CrIs).

\begin{tabular}{|c|c|c|c|c|c|c|c|c|c|}
\hline Endpoints & os & & & & & & & & \\
\hline \multirow{9}{*}{ PFS } & Placebo & $1.47(1.25,1.73)$ & $1.23(0.98,1.55)$ & $1.67(1.34,2.08)$ & $1.47(0.90,2.39)$ & $1.63(1.27,2.10)$ & $2.83(1.29,6.21)$ & $1.30(0.94,1.79)$ & $1.23(0.96,1.58)$ \\
\hline & $0.63(0.54,0.73)$ & TMZ & $0.84(0.64,1.11)$ & $1.14(0.98,1.32)$ & $1.00(0.60,1.67)$ & $1.11(0.91,1.35)$ & $1.92(0.89,4.15)$ & $0.88(0.62,1.26)$ & $0.84(0.62,1.13)$ \\
\hline & $0.65(0.53,0.80)$ & $1.03(0.80,1.34)$ & $\mathrm{PCV}$ & $1.35(0.99,1.86)$ & $1.19(0.70,2.04)$ & $1.32(0.94,1.85)$ & $2.29(1.01,5.20)$ & $1.05(0.71,1.56)$ & $1.00(0.90,1.11)$ \\
\hline & $0.40(0.32,0.50)$ & $0.64(0.55,0.75)$ & $0.62(0.46,0.84)$ & $\mathrm{BEV}+\mathrm{TMZ}$ & $0.88(0.52,1.50)$ & $0.97(0.76,1.24)$ & $1.69(0.77,3.71)$ & $0.78(0.53,1.14)$ & $0.74(0.53,1.03)$ \\
\hline & $0.75(0.49,1.15)$ & $1.19(0.75,1.88)$ & $1.15(0.71,1.86)$ & $1.86(1.15,3.02)$ & Nimotuzumab & $1.11(0.64,1.92)$ & $1.92(0.76,4.85)$ & $0.88(0.49,1.58)$ & $0.84(0.49,1.45)$ \\
\hline & $0.57(0.45,0.71)$ & $0.90(0.76,1.06)$ & $0.87(0.64,1.18)$ & $1.41(1.12,1.77)$ & $0.76(0.47,1.23)$ & Cilengitide $+\mathrm{TMZ}$ & $1.74(0.78,3.84)$ & $0.80(0.53,1.20)$ & $0.76(0.53,1.08)$ \\
\hline & $0.56(0.30,1.05)$ & $0.89(0.48,1.63)$ & $0.86(0.44,1.66)$ & $1.39(0.74,2.61)$ & $0.75(0.35,1.60)$ & $0.99(0.53,1.85)$ & $\mathrm{TMZ}+\mathrm{ACNU}+\mathrm{CDDP}$ & $0.46(0.20,1.07)$ & $0.44(0.19,1.00)$ \\
\hline & $0.75(0.57,0.98)$ & $1.19(0.87,1.62)$ & $1.15(0.81,1.62)$ & $1.86(1.31,2.64)$ & $1.00(0.60,1.67)$ & $1.32(0.93,1.88)$ & $1.34(0.68,2.65)$ & $\mathrm{DBD}+\mathrm{BCNU}$ & $0.95(0.63,1.42)$ \\
\hline & $0.65(0.52,0.82)$ & $1.03(0.78,1.36)$ & $1.00(0.91,1.10)$ & $1.62(1.18,2.23)$ & $0.87(0.53,1.42)$ & $1.15(0.83,1.59)$ & $1.17(0.60,2.27)$ & $0.87(0.61,1.25)$ & DMFO+PCV \\
\hline
\end{tabular}

intervention that significantly improved the PFS of glioma patients in comparison to the placebo $(\mathrm{HR}=0.65,95 \% \mathrm{CI}$ $=0.53-0.80)$. Apart from this, introducing BEV into TMZ improved the PFS of glioma patients dramatically by $36 \%(\mathrm{HR}=0.64,95 \% \mathrm{CI}=0.55-0.74)$. However, introducing other interventions into TMZ did not have considerable influence on the PFS of glioma patients (Cilengitide +TMZ vs. TMZ: HR $=0.90,95 \% \mathrm{CI}=0.76$ 1.06; TMZ+ACNU+CDDP $v s$. TMZ: $\mathrm{HR}=0.89,95 \% \mathrm{CI}$ $=0.49-1.62 ; \mathrm{DMFO}+\mathrm{PCV} v$ s. $\mathrm{PCV}: \mathrm{HR}=1.00,95 \% \mathrm{CI}=$ $0.91-1.11)$

\section{Network meta-analysis}

All mixed comparisons that synthesise both direct and indirect evidence are demonstrated in Table 3, Figure 3 and Figure 4. Placebo was proved to be significantly less effective than TMZ; BEV + TMZ; cilengitide + TMZ; TMZ, ACNU and CDDP. The corresponding HR and 95\% $\mathrm{CrI}$ for these comparisons were 1.47 with $95 \% \mathrm{CrI}$ 1.25 to $1.73,1.67$ with $95 \% \mathrm{CrI} 1.34$ to $2.08,1.63$ with $95 \%$ CrI 1.27 to 2.10 and 2.83 with $95 \%$ CrI 1.29 to 6.21 . Additionally, PCV showed less effective compared to the combined usage of TMZ, ACNU and CDDP: HR values amounted to 2.29 with $95 \% \mathrm{CrI}$ from 1.01 to 5.20. Apart 
from Nimotuzumab and TMZ + ACNU + CDDP, all other interventions were more effective than the placebo with respect to PFS of glioma patients. The corresponding HR values were listed as follows: TMZ with HR of 0.63 and $95 \% \mathrm{CrI}$ of 0.54 to $0.73, \mathrm{PCV}$ with HR of 0.65 and $95 \% \mathrm{CrI}$ of 0.53 to $0.80, \mathrm{BEV}+\mathrm{TMZ}$ with HR of 0.40 and $95 \% \mathrm{CrI}$ of 0.32 to 0.50 , cilengitide $+\mathrm{TMZ}$ with HR 0.57 and $95 \% \mathrm{CrI}$ of 0.45 to 0.71 , DBD + BCNU with HR of 0.75 and $95 \% \mathrm{CrI}$ of 0.57 to 0.98 and $\mathrm{DMFO}+\mathrm{PCV}$ with HR of 0.65 and $95 \% \mathrm{CrI}$ of 0.52 to 0.82 . Also, introducing $\mathrm{BEV}$ into TMZ significantly improved the PFS of glioma patients $(\mathrm{HR}=0.64,95 \% \mathrm{CrI}=0.55-0.75)$ and $\mathrm{PCV}$ remarkably improved the PFS of glioma patients $(\mathrm{HR}=$ $0.62,95 \% \mathrm{CrI}=0.46$ to 0.84 ). By contrast, nimotuzumab, cilengitide $+\mathrm{TMZ}, \mathrm{DBD}+\mathrm{BCNU}$ and $\mathrm{DMFO}+\mathrm{PCV}$ appeared to be less effective than BEV + TMZ and the corresponding HR for the above comparisons were: 1.86, $95 \% C r I=1.15-3.02 ; 1.41,95 \% C r I=1.12-1.77 ; 1.86$, $95 \% C r I=1.31-2.64$; and $1.62,95 \% C r I=1.18-2.23$.

The estimated ranking probabilities of each intervention calculated through SUCRA were illustrated in Figure 5. As suggested by the corresponding SUCRA, the intervention of TMZ + ACNU + CDDP was ranked as the most effective treatment combination with respect to OS whereas the intervention of $\mathrm{BEV}+\mathrm{TMZ}$ was ranked as the most effective one with respect to PFS. As a result, we performed a cluster analysis in order to simultaneously assess OS and PFS (Figure 6) and the corresponding treatments were categorized into three distinct clusters. The green cluster including BEV + TMZ, cilengitide + $\mathrm{TMZ}$ and $\mathrm{TMZ}+\mathrm{ACNU}+\mathrm{CDDP}$ appeared to have the most desirable OS and PFS whereas the blue cluster including nimotuzumab, TMZ and placebo seemed to be the least effective ones.

\section{DISCUSSION}

In this multiple treatment Bayesian meta-analysis, eight chemotherapy interventions in combination with RT after surgery were tested in this research. A total of 3,681 patients suffering from glioma were involved in this research and these patients were obtained from 14 randomized control trials or clinical trials. Also direct together with indirect evidence were taken into consideration in order to complete the conventional metaanalysis. The aim of this analysis was to examine the optimal chemotherapeutic treatment for glioma and to provide confidential guidance for clinical practice through assessing and comparing the prognosis of patients.

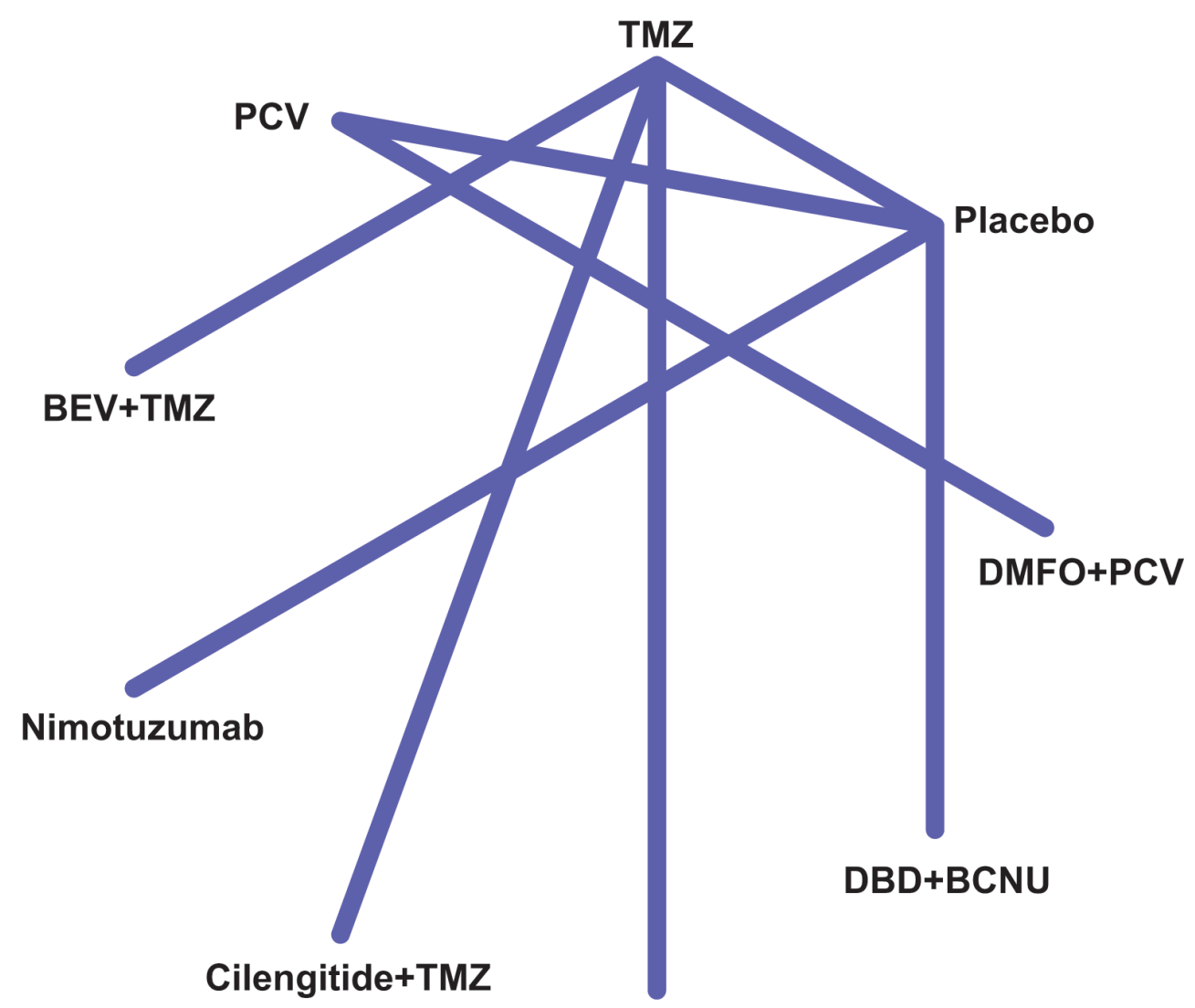

TMZ+ACNU+CDDP

Figure 2: Network of treatment strategies for glioma patients of included studies. 
As suggested by the rank probabilities of SUCRA, the combination of TMZ, ACNU and CDDP exhibited the most desirable OS. Another study conducted by Kim et al. [20] has identified the superiority of introducing both ACNU and CDDP into TMZ compared with single TMZ. Although TMZ, ACNU and CDDP were classified as alkylating agents, they had different mechanisms to control tumour cells. For instance, TMZ was converted into 5-(3-methyl)-1-triazen-1-yl-imidazole-4-carboximide (MTIC) through hydrolysis which provides affinity for guanine residues and induces either alkylation or methylation that further triggers apoptosis [33]. By contrast, ACNU is considered as the second-line chemotherapeutic intervention for glioma patients since it has comparable permeability for overcoming the bloodbrain barriers. However, ACNU functions through the formation of DNA monoalkylated adducts which may lead to spontaneous depuration, single-strand breaks or alkalilabile sites [34]. Although ACNU was prone to guanine residues, it is able to inhibit DNA synthesis by crosslinks [35]. However, damage caused by both TMZ and

\section{Treatment}

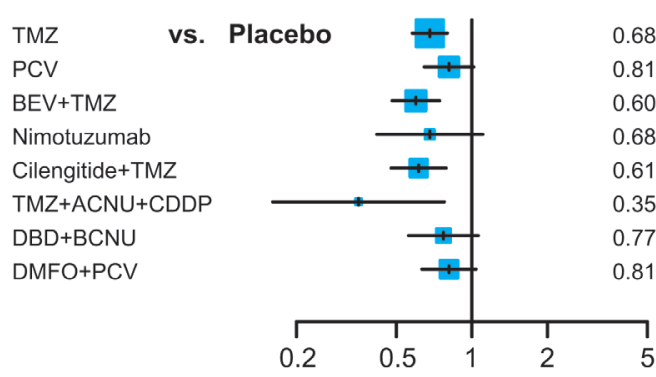

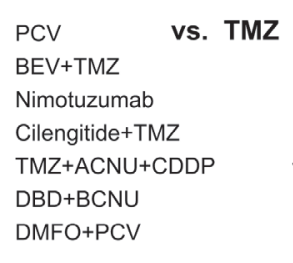
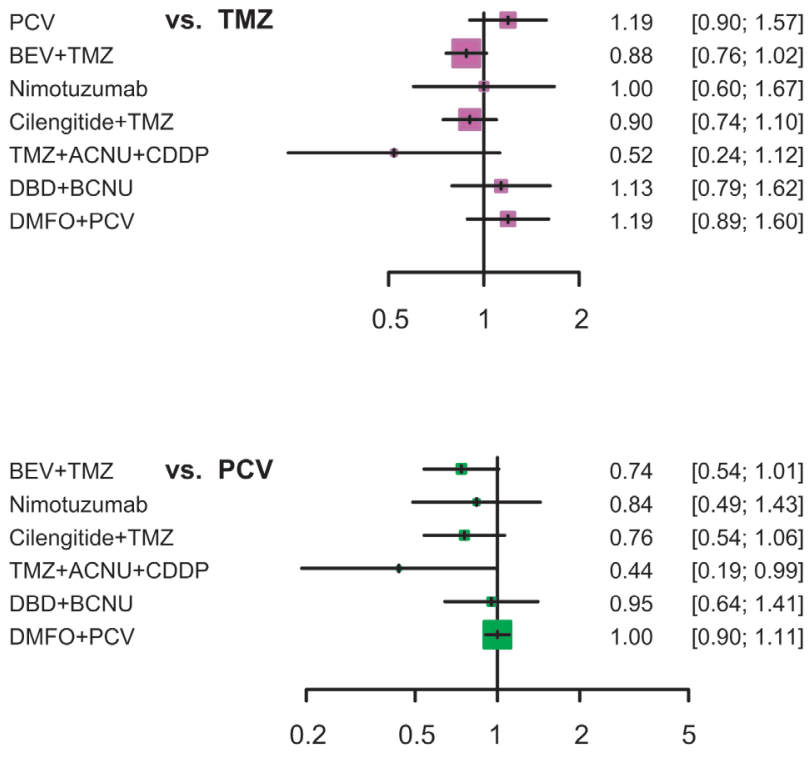

ACNU, especially the methylated product at O-6 position $\mathrm{O}^{6}$-methylguanine $\left(\mathrm{O}^{6} \mathrm{MeG}\right)$, can be restored by the DNA repair protein $\mathrm{O}^{6}$-methylguanine-DNA methyltransferase (MGMT) [36-38]. The presence of the MGMT enzyme prevented the apoptosis of tumour cells and suppressed the progression of both TMZ and ACNU [39]. On the other hand, CDDP can form multiple DNA adducts [40]. Unfortunately, this type of intervention is associated with several adverse effects. For instance, long term or high dosage of CDDP may induce cisplatin-resistant disease $[41,42]$ and it is acknowledged that CDDP has serious toxic effects such as ototoxicity and nephrotoxicity on patients [43]. Therefore, the combination of TMZ, ACNU and CDDP not only reduced their side effects on patients but also improved the prognosis of patients.

Unlike ACNU and CDDP, BEV is a humanized monoclonal antibody and it is the first angiogenesis inhibitor approved by the Food and Drug Administration (FDA). BEV is an antibody to VEGF-A, a predominant member of vascular endothelial growth factor (VEGF) family, which is highly expressed in tumour cells under
Treatment

$[0.58 ; 0.80]$ $[0.65 ; 1.02]$ $[0.48 ; 0.74]$ $[0.42 ; 1.11]$ $[0.48 ; 0.79]$ $[0.16 ; 0.78]$ $[0.56 ; 1.06]$ $[0.63 ; 1.04]$

$[0.90 ; 1.57]$ $[0.76 ; 1.02]$ $[0.60 ; 1.67]$ $[0.74 ; 1.10]$ $[0.24 ; 1.12]$ $[0.79 ; 1.62]$ $[0.89 ; 1.60]$ $\mathrm{DBD}+\mathrm{BCNU}$ DMFO+PCV
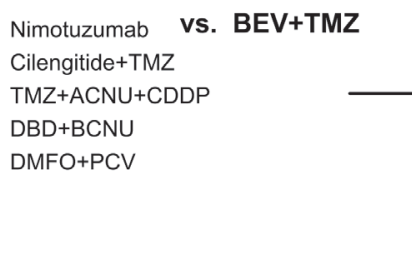

Cilengitide+TMZ vs. Nimotuzumab $T M Z+A C N U+C D D P$

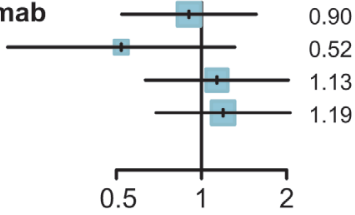

$[0.52 ; 1.56]$ $[0.21 ; 1.31]$ $[0.63 ; 2.03]$ $[0.69 ; 2.06]$

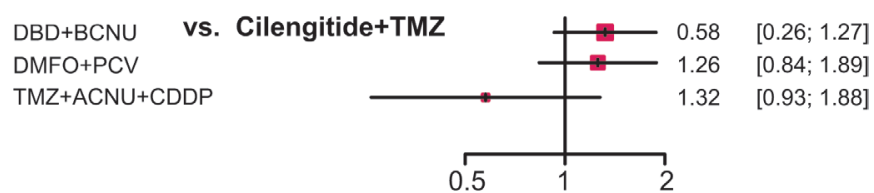

$\mathrm{DBD}+\mathrm{BCNU}$ $\mathrm{DMFO}+\mathrm{PCV}$

vs.

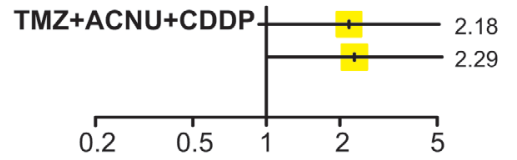

[0.93; 5.09] [1.00; 5.23]

DMFO+PCV

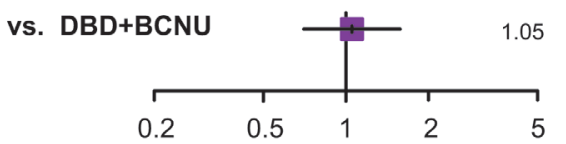

$[0.70 ; 1.58]$

Figure 3: Plot of the HR of OS for different treatment strategies from the network meta-analysis. 
hypoxia and could stimulate endothelial cell proliferation [44, 45]. Micro vessels regression, vessel growth and neovascularization inhibition can be achieved by antiVEGF treatment. Since vascular proliferation was one of the pathological hallmarks of GBM, continuous BEV is important in glioma treatment due to its inhibition of angiogenesis [46]. Nevertheless, single BEV in clinical practices has not been confirmed by researches due to its poor penetration through blood-brain barriers. As suggested by some hypothesises, BEV may cross the blood-brain barrier and reach its target if other drugs with strong permeability such as TMZ were introduced and this may explain the excellent performance of BEV combined with TMZ [47].

This Bayesian network meta-analysis evaluates eight popular chemotherapy interventions that were incorporated into RT for managing glioma patients in clinical practices. The OS and PFS of glioma patients were compared through synthesizing both direct and indirect evidence in order to overcome issues such as small sample size and lack of head-to-head comparisons. Nevertheless, some limitations should not be neglected due to the nature of network meta-analysis. For instance,

\section{Treatment}
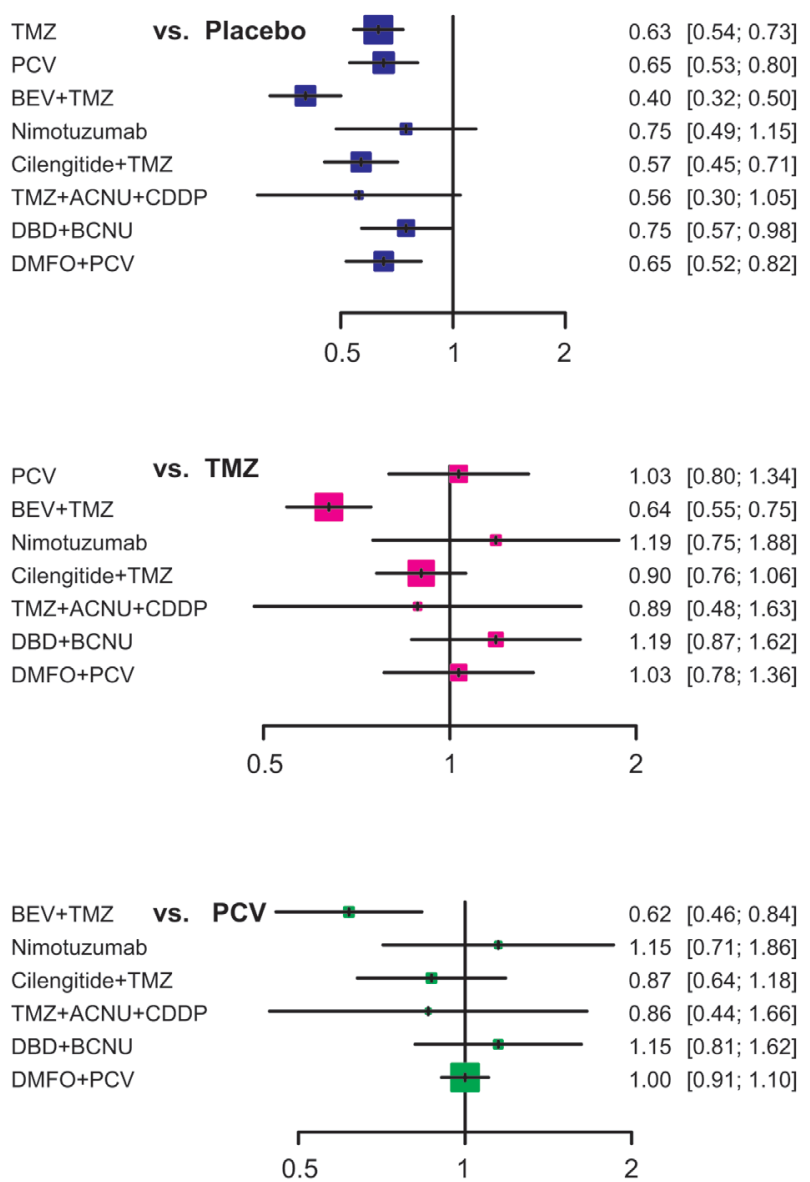

$0.40[0.32 ; 0.50]$ some chemotherapeutic interventions were intentionally excluded so that a closed loop of interventions can be formed for network meta-analysis. Besides that, five out of eight interventions-BEV + TMZ, nimotuzumab, $\mathrm{TMZ}+\mathrm{ACNU}+\mathrm{CDDP}, \mathrm{DBD}+\mathrm{BCNU}, \mathrm{DMFO}+\mathrm{PCV}-$ ontained only one eligible study and such a trend could have significant influence on the overall conclusions. Furthermore, factors such as different strategies of RT and the extent of surgery in each study were completely ignored and it is likely that the overall effectiveness of these chemotherapeutic may vary with the above mentioned confounding factors. Finally, our study did not take the modality of glioma into account and such an issue should be investigated by future researchers.

This study provided exclusive evidence that some chemotherapeutic agents including ACNU, CDDP or $\mathrm{BEV}$ can be introduced into TMZ in order to enhance its efficacy and hence improve the survival status of glioma patients. However, clinicians should be attentive to the characteristics of patients as well as the contraindications of chemotherapeutic interventions when selecting the most appropriate one for glioma patients.

\section{Treatment}

$0.63[0.54 ; 0.73]$

$0.65[0.53 ; 0.80]$

$0.75[0.49 ; 1.15]$

$0.57[0.45 ; 0.71]$

$0.56[0.30 ; 1.05]$

$0.75[0.57 ; 0.98]$

$0.65[0.52 ; 0.82]$
HR 95\%Cl

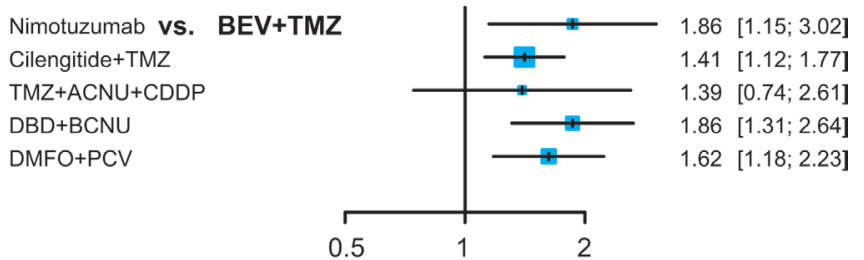

DMFO+PCV vs. Nimotuzumab $\mathrm{TMZ}+\mathrm{ACNU}+\mathrm{CDDP}$

$\mathrm{DBD}+\mathrm{BCNU}$

Cilengitide $+\mathrm{TMZ}$

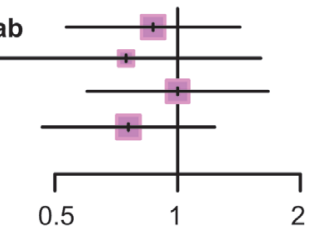

$0.76[0.47 ; 1.23]$

$0.75[0.35 ; 1.60]$

$1.00[0.60 ; 1.67]$

$0.87[0.53 ; 1.42]$
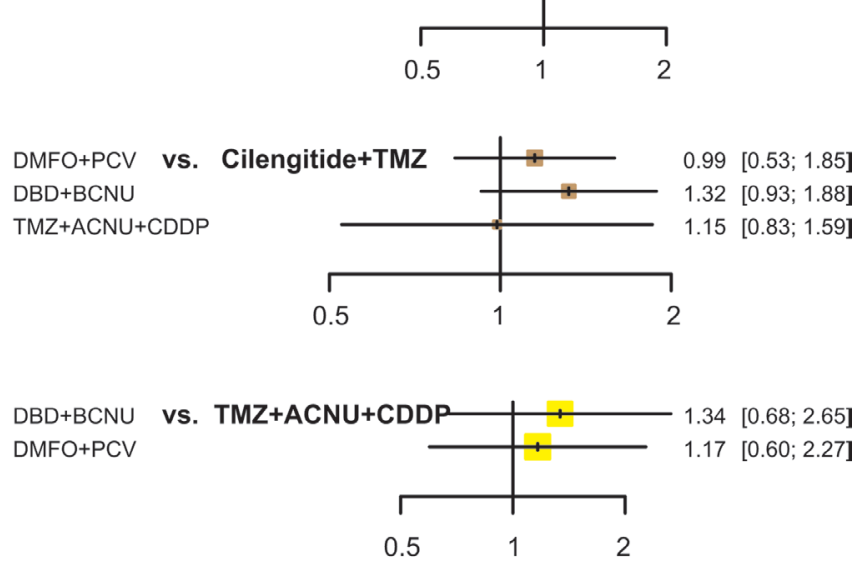

$\mathrm{DMFO}+\mathrm{PCV}$ vs. $\mathrm{DBD}+\mathrm{BCNU}$

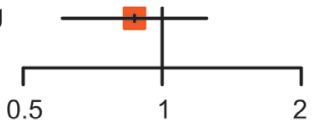

$0.87[0.61 ; 1.25]$

Figure 4: Plot of the HR of PFS for different treatment strategies from the network meta-analysis. 
A OS
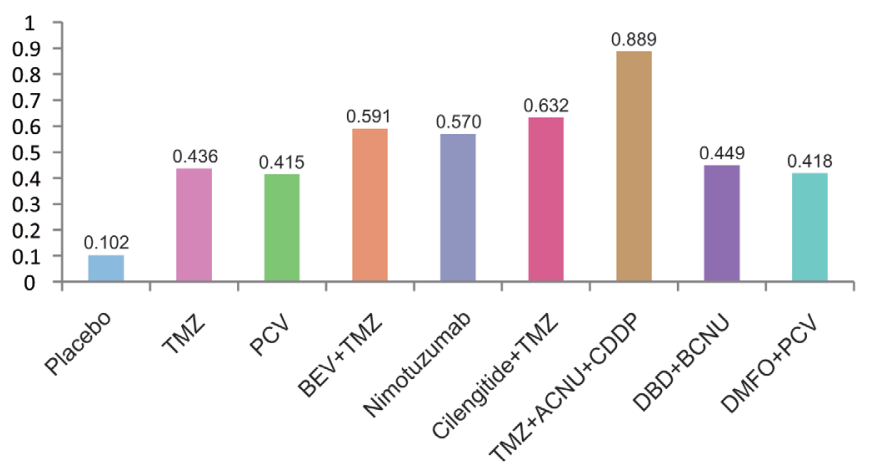

B PFS

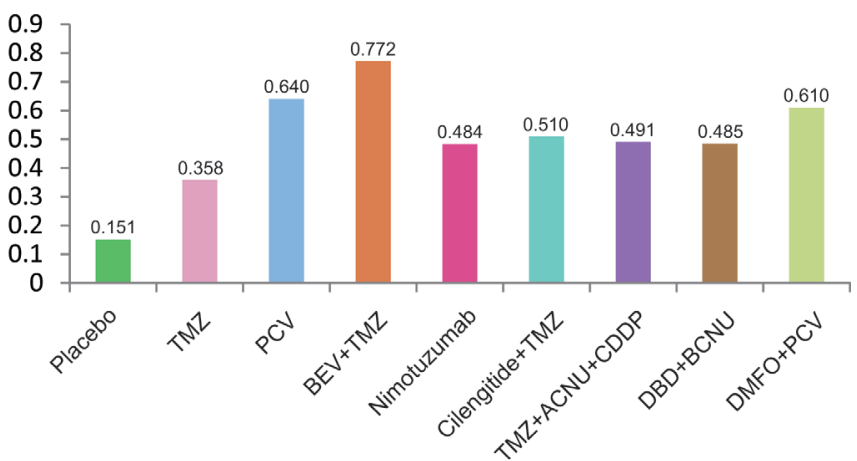

Figure 5: The cumulative ranking probabilities of different treatment strategies of OS and PFS.

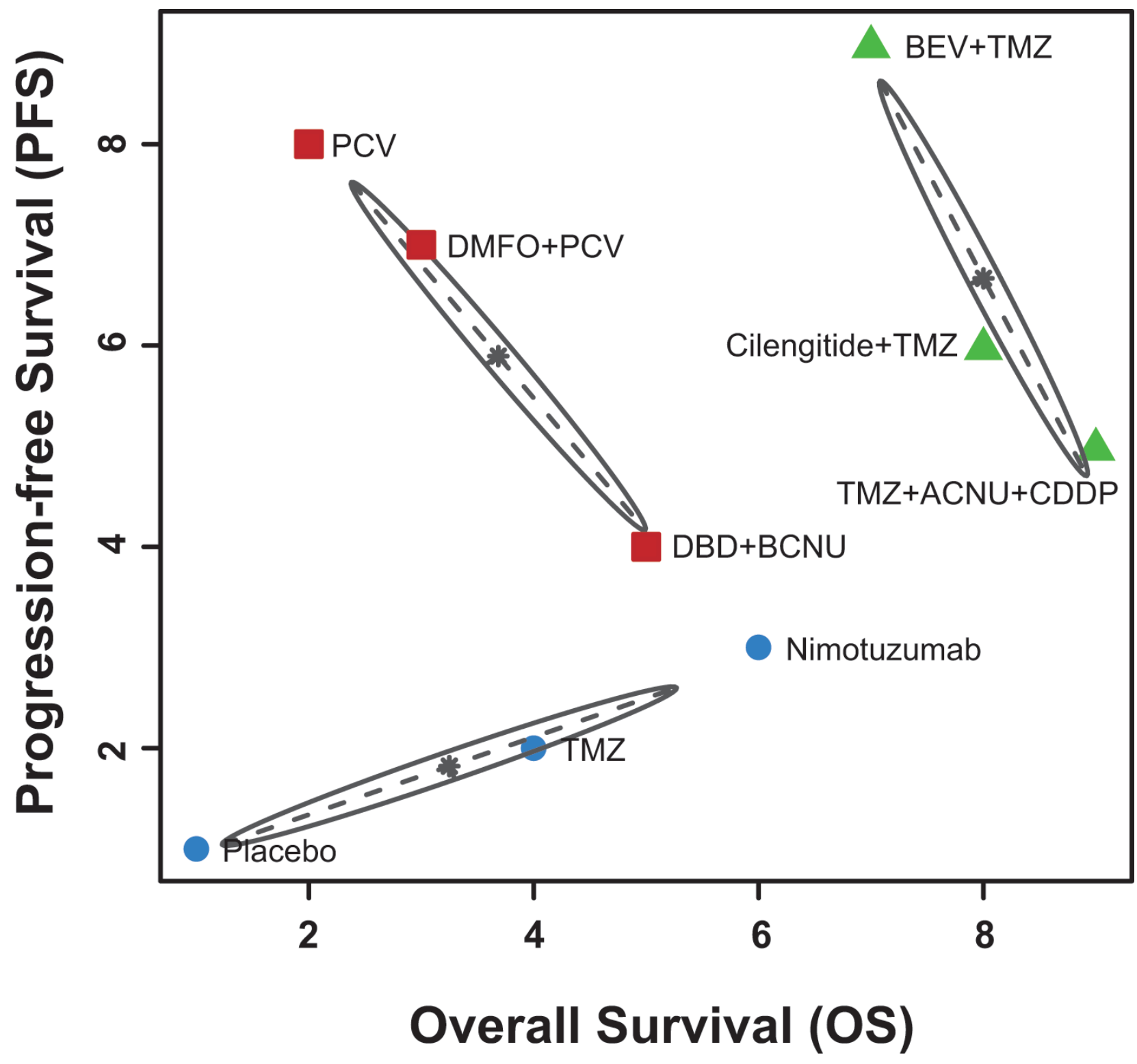

Figure 6: The cluster analysis of different treatment strategies of OS and PFS. 


\section{MATERIALS AND METHODS}

\section{Search strategy}

Medical databases including PubMed, Embase and Cochrane Library were consulted to identify all eligible randomized control trials that were not restricted by particular languages. The following searching terms with their corresponding synonyms were used to find relevant literatures: "glioma", "radiotherapeutic treatment", "surgery", "chemotherapeutic agents", "clinical trial" and "randomized control trial". The reference list of every article was examined manually in order to prevent relevant articles being excluded and two reviewers (Ling Qi and Lijuan Ding) retrieved all the potential literature independently.

\section{Inclusion and exclusion criteria}

Studies were eligible if they satisfied the following criteria: (i) they were randomized control trials, or clinical trials in phase II or III with more than 30 subjects; (ii) all the subjects were adults who were diagnosed with glioma, irrespective of different glioma types or grades; (iii) all chemotherapy drugs which were studied must be used with RT after surgery, although there was no restriction on the type of RT or the scope of surgery; (iv) studies must investigate the OS and PFS of glioma patients treated with at least two chemotherapeutic treatments or placebos. Additionally, even though the criteria above were met, studies were eliminated if they satisfied any exclusion criteria: (i) examined only one chemotherapy drug for which different delivery methods were compared such as intravenous and intra-arterial administration [13]; (ii) the reported interventions were not within a closed loop since indirect comparisons between two interventions cannot be established by linking them with a third intervention. The title and abstract of all the retrieved literatures were screened, and full text was also examined for the sake of determining the eligibility of studies. Two reviewers (Ling Qi and Lijuan Ding) performed all the above procedures independently and disagreement was resolved by discussion.

\section{Outcome measures and data extraction}

For each eligible study, the following information was extracted: the main characteristics of the study including author; year of publication; country; type of study; sample size; baseline characteristics of patients such as glioma modality, newly or recurrent glioma, extent of surgery, type of RT, intervention details such as delivery methods and doses. Apart from these, interventions were evaluated using outcomes including median OS time, HR of OS, median time of PFS, HR of PFS as well as the number of adverse events occurred in each group.

Network meta-analysis (NMA) has been designed to compare the prognosis of glioma patients treated by different chemotherapeutic agents and primary endpoints incorporated in NMA were the OS and PFS for which the median value as well as the HR were compared. As defined by the National Cancer Institute (NIH), the OS time is the time between treatment commencements and patient death. OS is considered as a standard primary endpoint which is easy and precise to assess the overall effectiveness of interventions in oncologic clinical trials [14]. On the other hand, PFS is defined between the time at which disease was not progressed and the time of patient death. Both OS and PFS provide comprehensive evidence for the effectiveness of a specific chemotherapy. For those studies in which data of OS or PFS were not presented, we estimated these figures either using the cumulative survival percentages or the available survival curves.

\section{Statistical analysis}

We set the significance level at $\alpha=0.05$ for all statistical tests. Heterogeneity between studies were assessed by the Cochran's $Q$ statistic which calculates the weighted sum of squared differences [15] as:

$$
Q=\sum \omega_{i}\left(\theta_{i}-\theta\right)^{2}
$$

The proportion of the observed variance contributed by real differences in effect sizes rather than sampling errors was measured by the statistic of $I^{2}[16]$ :

$$
I^{2}=\frac{Q-(k-1)}{Q} \times 100 \% \text {, where } k=\text { the number of studies }
$$

In this case, the random-effects model was more appropriate than the fixed-effect model [17]. Subsequently, a NMA was conducted for both OS and PFS based on a Bayesian framework using R 3.2.3 software. Both direct and indirect evidence were synthesized in the NMA and the results were described as ORs with their corresponding 95\% credible intervals. Furthermore, the surface under the cumulative ranking curve (SUCRA) was adopted to identify the optimal interventions with respect to different endpoints. The probabilities that each intervention ranks the best, second best and third best etc., were calculated in percentages and then these numerical values were accumulated in order to obtain their corresponding SUCRA values. All the investigated treatments were ranked based on their corresponding SUCRA values and a higher value of SUCRA provided evidence for more desirable OS and PFS time [18]. In addition, a cluster analysis was performed to group similar interventions by combining different endpoints. 


\section{ACKNOWLEDGMENTS}

This work is supported by National Natural Science Foundation of China (No. 81201671; 81372793) and Foundation of Science and Technology Department of Jilin Province (No. 20150414034GH; 20140414049GH; 20160101195JC).

\section{CONFLICTS OF INTEREST}

The authors declare no conflict of interest.

\section{REFERENCES}

1. Jessen KR and Mirsky R. Glial cells in the enteric nervous system contain glial fibrillary acidic protein. Nature. 1980; 286:736-737.

2. Goodenberger ML and Jenkins RB. Genetics of adult glioma. Cancer genetics. 2012; 205:613-621.

3. Reuss D and von Deimling A. Hereditary tumor syndromes and gliomas. Recent results in cancer research Fortschritte der Krebsforschung Progres dans les recherches sur le cancer. 2009; 171:83-102.

4. Louis DN, Ohgaki H, Wiestler OD, Cavenee WK, Burger PC, Jouvet A, Scheithauer BW and Kleihues P. The 2007 WHO classification of tumours of the central nervous system. Acta neuropathologica. 2007; 114:97-109.

5. Schwartzbaum JA, Fisher JL, Aldape KD and Wrensch M. Epidemiology and molecular pathology of glioma. Nature clinical practice Neurology. 2006; 2:494-503; quiz 491 p following 516.

6. Ohgaki H, Dessen P, Jourde B, Horstmann S, Nishikawa T, Di Patre PL, Burkhard C, Schuler D, Probst-Hensch NM, Maiorka PC, Baeza N, Pisani P, Yonekawa Y, Yasargil MG, Lutolf UM and Kleihues P. Genetic pathways to glioblastoma: a population-based study. Cancer research. 2004; 64:6892-6899.

7. Vecht CJ, Avezaat CJ, van Putten WL, Eijkenboom WM and Stefanko SZ. The influence of the extent of surgery on the neurological function and survival in malignant glioma. A retrospective analysis in 243 patients. Journal of neurology, neurosurgery, and psychiatry. 1990; 53:466-471.

8. Stummer W, Reulen HJ, Meinel T, Pichlmeier U, Schumacher W, Tonn JC, Rohde V, Oppel F, Turowski B, Woiciechowsky C, Franz K, Pietsch T and Group AL-GS. Extent of resection and survival in glioblastoma multiforme: identification of and adjustment for bias. Neurosurgery. 2008; 62:564-576; discussion 564-576.

9. Kuhnt D, Becker A, Ganslandt O, Bauer M, Buchfelder M and Nimsky C. Correlation of the extent of tumor volume resection and patient survival in surgery of glioblastoma multiforme with high-field intraoperative MRI guidance. Neuro-oncology. 2011; 13:1339-1348.

10. Beez T, Boge K, Wager M, Whittle I, Fontaine D, Spena
G, Braun S, Szelenyi A, Bello L, Duffau H, Sabel M and European Low Grade Glioma N. Tolerance of awake surgery for glioma: a prospective European Low Grade Glioma Network multicenter study. Acta neurochirurgica. 2013; 155:1301-1308.

11. Barani IJ and Larson DA. Radiation therapy of glioblastoma. Cancer treatment and research. 2015; 163:4973.

12. Omuro A and DeAngelis LM. Glioblastoma and other malignant gliomas: a clinical review. Jama. 2013; 310:18421850.

13. Imbesi F, Marchioni E, Benericetti E, Zappoli F, Galli A, Corato $\mathrm{M}$ and Ceroni M. A randomized phase III study: comparison between intravenous and intraarterial ACNU administration in newly diagnosed primary glioblastomas. Anticancer research. 2006; 26:553-558.

14. Driscoll JJ and Rixe O. Overall survival: still the gold standard: why overall survival remains the definitive end point in cancer clinical trials. Cancer journal. 2009; 15:401405.

15. Higgins JP and Thompson SG. Quantifying heterogeneity in a meta-analysis. Statistics in medicine. 2002; 21:1539-1558.

16. Higgins JP, Thompson SG, Deeks JJ and Altman DG. Measuring inconsistency in meta-analyses. Bmj. 2003; 327:557-560.

17. Ades AE, Lu G and Higgins JP. The interpretation of random-effects meta-analysis in decision models. Med Decis Making. 2005; 25:646-654.

18. Rucker $G$ and Schwarzer G. Ranking treatments in frequentist network meta-analysis works without resampling methods. BMC medical research methodology. 2015; 15:58.

19. Stupp R, Hegi ME, Gorlia T, Erridge SC, Perry J, Hong YK, Aldape KD, Lhermitte B, Pietsch T, Grujicic D, Steinbach JP, Wick W, Tarnawski R, Nam DH, Hau P, Weyerbrock A, et al. Cilengitide combined with standard treatment for patients with newly diagnosed glioblastoma with methylated MGMT promoter (CENTRIC EORTC 26071-22072 study): a multicentre, randomised, open-label, phase 3 trial. The Lancet Oncology. 2014; 15:1100-1108.

20. Kim IH, Park CK, Heo DS, Kim CY, Rhee CH, Nam DH, Lee SH, Han JH, Lee SH, Kim TM, Kim DW, Kim JE, Paek SH, Kim DG, Kim IA, Kim YJ, et al. Radiotherapy followed by adjuvant temozolomide with or without neoadjuvant ACNU-CDDP chemotherapy in newly diagnosed glioblastomas: a prospective randomized controlled multicenter phase III trial. Journal of neurooncology. 2011; 103:595-602.

21. van den Bent MJ, Carpentier AF, Brandes AA, Sanson M, Taphoorn MJ, Bernsen HJ, Frenay M, Tijssen CC, Grisold W, Sipos L, Haaxma-Reiche H, Kros JM, van Kouwenhoven MC, Vecht CJ, Allgeier A, Lacombe D, et al. Adjuvant procarbazine, lomustine, and vincristine improves progression-free survival but not overall survival 
in newly diagnosed anaplastic oligodendrogliomas and oligoastrocytomas: a randomized European Organisation for Research and Treatment of Cancer phase III trial. J Clin Oncol. 2006; 24:2715-2722.

22. Nabors LB, Fink KL, Mikkelsen T, Grujicic D, Tarnawski R, Nam do H, Mazurkiewicz M, Salacz M, Ashby L, Zagonel V, Depenni R, Perry JR, Hicking C, Picard M, Hegi ME, Lhermitte B, et al. Two cilengitide regimens in combination with standard treatment for patients with newly diagnosed glioblastoma and unmethylated MGMT gene promoter: results of the open-label, controlled, randomized phase II CORE study. Neuro-oncology. 2015; 17:708-717.

23. Kocher M, Frommolt P, Borberg SK, Ruhl U, Steingraber M, Niewald M, Staar S, Stuschke M, Becker G, Fischedick AR, Herfarth K, Grauthoff $\mathrm{H}$ and Muller RP. Randomized study of postoperative radiotherapy and simultaneous temozolomide without adjuvant chemotherapy for glioblastoma. Strahlenther Onkol 2008; 184:572-579.

24. Muni R, Minniti G, Lanzetta G, Caporello P, Frati A, Enrici MM, Marchetti P and Enrici RM. Short-term radiotherapy followed by adjuvant chemotherapy in poor-prognosis patients with glioblastoma. Tumori. 2010; 96:60-64.

25. Chinot OL, Wick W, Mason W, Henriksson R, Saran F, Nishikawa R, Carpentier AF, Hoang-Xuan K, Kavan P, Cernea D, Brandes AA, Hilton M, Abrey L and Cloughesy T. Bevacizumab plus radiotherapy-temozolomide for newly diagnosed glioblastoma. The New England journal of medicine. 2014; 370:709-722.

26. Hildebrand J, Gorlia T, Kros JM, Afra D, Frenay M, Omuro A, Stupp R, Lacombe D, Allgeier A and van den Bent MJ. Adjuvant dibromodulcitol and BCNU chemotherapy in anaplastic astrocytoma: results of a randomised European Organisation for Research and Treatment of Cancer phase III study (EORTC study 26882). European journal of cancer (Oxford, England : 1990). 2008; 44:1210-1216.

27. Combs SE, Nagy M, Edler L, Rausch R, Bischof M, Welzel T, Debus J and Schulz-Ertner D. Comparative evaluation of radiochemotherapy with temozolomide versus standardof-care postoperative radiation alone in patients with WHO grade III astrocytic tumors. Radiother Oncol. 2008; 88:177182.

28. Shaw EG, Wang M, Coons SW, Brachman DG, Buckner JC, Stelzer KJ, Barger GR, Brown PD, Gilbert MR and Mehta MP. Randomized trial of radiation therapy plus procarbazine, lomustine, and vincristine chemotherapy for supratentorial adult low-grade glioma: initial results of RTOG 9802. J Clin Onco. 2012; 30:3065-3070.

29. Tham CK, See SJ, Tan SH, Lim KH, Ng WH, Thomas J, Chong DQ and Chua ET. Combined temozolomide and radiation as an initial treatment for anaplastic glioma. AsiaPacific journal of clinical oncology. 2013; 9:220-225.

30. Levin VA, Uhm JH, Jaeckle KA, Choucair A, Flynn PJ, Yung WKA, Prados MD, Bruner JM, Chang SM, Kyritsis AP, Gleason MJ and Hess KR. Phase III randomized study of postradiotherapy chemotherapy with alpha- difluoromethylornithine-procarbazine, N-(2-chloroethyl)N'-cyclohexyl-N-nitrosurea, vincristine (DFMO-PCV) versus PCV for glioblastoma multiforme. Clin Cancer Res. 2000; 6:3878-3884.

31. Solomon MT, Selva JC, Figueredo J, Vaquer J, Toledo C, Quintanal N, Salva S, Domingez R, Alert J, Marinello JJ, Catala M, Griego MG, Martell JA, Luaces PL, Ballesteros $\mathrm{J}$, de-Castro N, et al. Radiotherapy plus nimotuzumab or placebo in the treatment of high grade glioma patients: results from a randomized, double blind trial. BMC cancer. 2013; 13:299.

32. Stupp R, Mason WP, van den Bent MJ, Weller M, Fisher B, Taphoorn MJ, Belanger K, Brandes AA, Marosi C, Bogdahn U, Curschmann J, Janzer RC, Ludwin SK, Gorlia T, Allgeier A, Lacombe D, et al. Radiotherapy plus concomitant and adjuvant temozolomide for glioblastoma. The New England journal of medicine. 2005; 352:987-996.

33. Mrugala MM and Chamberlain MC. Mechanisms of disease: temozolomide and glioblastoma-look to the future. Nature clinical practice Oncology. 2008; 5:476-486.

34. Zhao LN, Chen XC, Zhong YY, Hou QX and Zhong RG. Nimustine Induces DNA Breaks and Crosslinks in NIH/3T3 Cells. International Journal of Bioscience Biochemistry \& Bioinformatics. 2013; 3.

35. Agarwal S, Ray B and Mehrotra R. SERS as an advanced tool for investigating chloroethyl nitrosourea derivatives complexation with DNA. International journal of biological macromolecules. 2015; 81:891-897.

36. Goth R and Rajewsky MF. Persistence of O6-ethylguanine in rat-brain DNA: correlation with nervous system-specific carcinogenesis by ethylnitrosourea. Proceedings of the National Academy of Sciences of the United States of America. 1974; 71:639-643.

37. Kaina B, van Zeeland AA, de Groot A and Natarajan AT. DNA repair and chromosomal stability in the alkylating agent-hypersensitive Chinese hamster cell line 27-1. Mutation research. 1990; 243:219-224.

38. Kondo N, Takahashi A, Ono K and Ohnishi T. DNA damage induced by alkylating agents and repair pathways. Journal of nucleic acids. 2010; 2010:543531.

39. Roos WP, Batista LF, Naumann SC, Wick W, Weller M, Menck CF and Kaina B. Apoptosis in malignant glioma cells triggered by the temozolomide-induced DNA lesion O6-methylguanine. Oncogene. 2007; 26:186-197.

40. Zamble DB and Lippard SJ. Cisplatin and DNA repair in cancer chemotherapy. Trends in biochemical sciences. 1995; 20:435-439.

41. Stordal B and Davey M. Understanding cisplatin resistance using cellular models. IUBMB life. 2007; 59:696-699.

42. Wang D and Lippard SJ. Cellular processing of platinum anticancer drugs. Nature reviews Drug discovery. 2005; 4:307-320.

43. Ravi R, Somani SM and Rybak LP. Mechanism of cisplatin ototoxicity: antioxidant system. Pharmacology \& 
toxicology. 1995; 76:386-394.

44. Los M, Roodhart JM and Voest EE. Target practice: lessons from phase III trials with bevacizumab and vatalanib in the treatment of advanced colorectal cancer. The oncologist. 2007; 12:443-450.

45. Mukherji SK. Bevacizumab (Avastin). AJNR American journal of neuroradiology. 2010; 31:235-236.
46. Norden AD, Young GS, Setayesh K, Muzikansky A, Klufas R, Ross GL, Ciampa AS, Ebbeling LG, Levy B, Drappatz J, Kesari S and Wen PY. Bevacizumab for recurrent malignant gliomas: efficacy, toxicity, and patterns of recurrence. Neurology. 2008; 70:779-787.

47. Iwamoto FM and Fine HA. Bevacizumab for malignant gliomas. Archives of neurology. 2010; 67:285-288. 